Supporting Information

\title{
Site-Specific Fragmentation of Green Fluorescent Protein Induced by Blue Light
}

Philipp J. Heckmeier ${ }^{\mathrm{a}, \mathrm{b}}$ and Dieter Langosch ${ }^{\mathrm{a}, *}$

a Lehrstuhl für Chemie der Biopolymere, Technische Universität München, Weihenstephaner Berg 3, 85354 Freising, Germany

${ }^{\mathrm{b}}$ present address: Department of Chemistry, University of Zürich. Winterthurerstrasse 190, CH-8057 Zürich, Switzerland

* corresponding author

e-mail: langosch@tum.de

Contents:

Materials and Methods

Supporting Figures $\mathrm{S}_{1}, \mathrm{~S}_{2}, \mathrm{~S}_{3}, \mathrm{~S}_{4}$, and $\mathrm{S}_{\mathbf{5}}$

Supporting References 


\section{Materials and Methods}

\section{Cloning, protein expression, and protein purification}

In order to construct the sfGFP fusion protein, a synthetic reading frame (BioCat, Heidelberg, Germany) was inserted into a pET28a plasmid backbone (Novagen, Merck, Darmstadt, Germany). The reading frame contained a sfGFP domain, an N-terminal Nano-tag and a Cterminal FLAG-tag for western blot detection, glycine/serine-based flexible linker regions, and a polyhistidine-tag at the C-terminus for protein purification as described previously ${ }^{1}$. The plasmid with EGFP was a kind gift from Ajitha Cristie-David and Neil Marsh, University of Michigan, Ann Arbor, MI ${ }^{2}$. The proteins were expressed in Escherichia coli BL21 (DE3) and purified via immobilized metal affinity chromatography as described ${ }^{~}$. After the purification, the samples were dialyzed against flow cytometry grade PBS ( $\mathrm{pH} 7.4$; Thermo Fisher Scientific, Waltham, MA, USA) containing $1 \mathrm{mM}$ EDTA, $0.5 \mathrm{mM} \beta$-mercaptoethanol, and $0.05 \%(\mathrm{v} / \mathrm{v})$ sodium azide. The concentration of the GFP fusion proteins was determined by UV-VIS specroscopy using an extinction coefficient of $83,300 \mathrm{~cm}^{-1} \mathrm{M}^{-1}$ (sfGFP) and 55,900 $\mathrm{cm}^{-1} \mathrm{M}^{-1}$ (EGFP). The quantum yield of sfGFP (o.7o) is similar to the previously reported one $(=0.65)^{3}$ thus demonstrating its fully folded conformation ${ }^{1}$.

\section{Irradiation of the GFP Samples}

GFP samples of $2 \mu \mathrm{M}$ in $1 \mathrm{~mL}$ of dialysis buffer were irradiated with a $445 \mathrm{~nm}$ laser diode (PM120, Thorlabs, Newton, NJ, USA; settings: $0.45 \mathrm{~A}, 5.5 \mathrm{~V}$ ) in successive intervalls of 10minutes at a pathlength of $2 \mathrm{~cm}$ with the laser diode placed $\sim 3 \mathrm{~cm}$ above the cuvette (OD488 $\approx 0.2$ ). The samples were kept in a plastic cuvette that was encased by aluminium foil and water-cooled to $\sim 6^{\circ} \mathrm{C}$. The volume was not stirred during the treatment. Unless otherwise specified, the laser output was $306 \mathrm{~mW}$ as determined previously ${ }^{1}$. At this laser power, the power density is $15 \mathrm{~kW} / \mathrm{m}^{2}$ which corresponds to a light dose of $1.75{ }^{*} 10^{7} \mathrm{~J} / \mathrm{m}^{2}$ per hour of irradiation at $300 \mathrm{~mW}^{1}$. Under our experimental conditions, a theoretical amount of 18300 absorbed photons per GFP molecule per minute was calculated.

\section{Fluorescence Spectroscopy}

To record the fluorescence emission spectrum of the sfGFP fusion protein, the samples were excited at $\lambda_{\mathrm{ex}}=488 \mathrm{~nm}$ in a $10 \mathrm{~mm}$ precision quartz cuvette (Hellma Analytics, Müllheim, Germany). The spectrum was recorded with a Cary Eclipse fluorimeter (Varian, Darmstadt, Germany). Fluorescence intensity measurements were executed with a FLUOstar microplate reader (BMG Labtech, Ortenberg, Germany) at $\lambda_{\mathrm{ex}}=488 \mathrm{~nm} ; \lambda_{\mathrm{em}}=520 \mathrm{~nm}$.

\section{SDS-PAGE Analysis and Immunoblotting}

Protein samples were analyzed via SDS PAGE. For gels without a pore size gradient, the separating and stacking gels contained $10 \%$ or $5 \%(\mathrm{v} / \mathrm{v})$ acrylamide-bisacrylamide, respectively. Gradient gels consisted of three different layers, a small-pore $16.5 \%(\mathrm{v} / \mathrm{v})$, the large-pore $10 \%$ $(\mathrm{v} / \mathrm{v})$, and a stacking $4 \%(\mathrm{v} / \mathrm{v})$ gel 4 . Prior to loading, protein samples in SDS sample buffer were boiled for $10 \mathrm{~min}$ at $95{ }^{\circ} \mathrm{C}$. The gels were stained with Coomassie Brilliant Blue G250 
solution. Protein bands were analyzed via densitometry with ImageStudio Lite (LI COR, Lincoln, NE, USA).

To generate Figures $2 \mathrm{~B}$ and $3 \mathrm{~B}$, we used SDS-PAGE to analyze protein samples which were irradiated for different periods of time. Prior to running the gels, we recorded the remaining fluorescence of these samples (yielding "fraction of inactivated fluorophores", x-axis). After running the gel, we determined the staining intensity of protein bands of interest by densitometry, normalized to the intensity of the respective full-length protein band (yielding "intact protein band strength" for Fig $2 \mathrm{~B}$, or "normalized staining intensity" for Fig $3 \mathrm{~B}$, yaxis)."The densitometric analysis of the protein bands was executed with three independent gels for sfGFP, and one gel for EGFP.

For immunoblot analysis, irradiated and non-irradiated sfGFP were first run on an SDS-PAGE gel as described above. In a semi-dry blotting procedure, the proteins were transferred from the gel onto a nitrocellulose membrane (Berrytec, Grünwald, Germany). To check the transfer efficiency, the membrane was stained with Ponceau $S$. The proteins were detected by primary antibodies specifically binding Nano-tag (DVEAWLGAR, MyBioSource, CA, US) or FLAG-tag (YKGDYKDHDG; Sigma-Aldrich, St. Louis, MO, US) and a secondary antibody fused to an alkaline phosphatase (AP). Finally, the protein bands were visualized via 5-bromo-4-chloro-3indolyl phosphate (BCIP) and p-nitroblue tetrazolium chloride (NBT) staining.

\section{Mass Spectrometric (MS) Analysis}

Matrix-assisted laser desorption/ionization (MALDI)-MS. For sample preparation, the purified protein solution ( $15 \mu \mathrm{M}$ in PBS) was mixed with an equal volume of a saturated solution of sinapinic acid (30\% acetonitrile, $0.42 \%$ TFA) and $1 \mu \mathrm{L}$ was spotted on a stainlesssteel MALDI target plate (Sciex). The protein fragments were then measured in a 4800 MALDI TOF-TOF instrument (Sciex) operated in linear high mass positive mode with an accelerating voltage of $25 \mathrm{kV}$ in a mass range between 5.000-40.000 (Focus Mass: 30.000). For each spectrum 500 shots were recorded and averaged.

Electrospray ionization (ESI)-MS. ESI-MS data were acquired on a Synapt G2-Si quadrupole time-of-flight spectrometer (Waters, UK) with a capillary voltage of $3 \mathrm{~V}$, a cone voltage of $50 \mathrm{~V}$, and a source temperature of $80{ }^{\circ} \mathrm{C}$. Prior ESI-MS analysis, samples were acidified with $1 \%$ formic acid (Thermo, USA), desalted using $\mathrm{C}_{4}$ Zip Tips (Millipore, USA) and analyzed in methanol:2-propanol:0.2\% formic acid (30:20:50). Solution was infused through a fused silica capillary (ID75um) at a flow rate of 1 uLmin-1 and sprayed through a Pico Tips (IDzoum). The last were obtained from New Objective (Woburn, MA). Mass spectra were acquired in the positive-ion mode by scanning an $\mathrm{m} / \mathrm{z}$ range from 400 to 4000 da with a scan duration of $1 \mathrm{~s}$ and an interscan delay of o.1s. The spray voltage was set to $3 \mathrm{kV}$, the cone voltage to $50 \mathrm{~V}$, and source temperature $80{ }^{\circ} \mathrm{C}$. Recorded $\mathrm{m} / \mathrm{z}$ data were deconvoluted using the MaxEntı software with a resolution of the output mass of 0.5 Da per channel and a Uniform Gaussian Damage Model at the half-height of $0.5 \mathrm{Da}$. The monoisotopic masses of the smaller fragments were deconvoluted with a resolution of throughput mass $0.03 \mathrm{Da} /$ channel and Uniform Gaussian Damage Model at the half height of o.1 Da. 


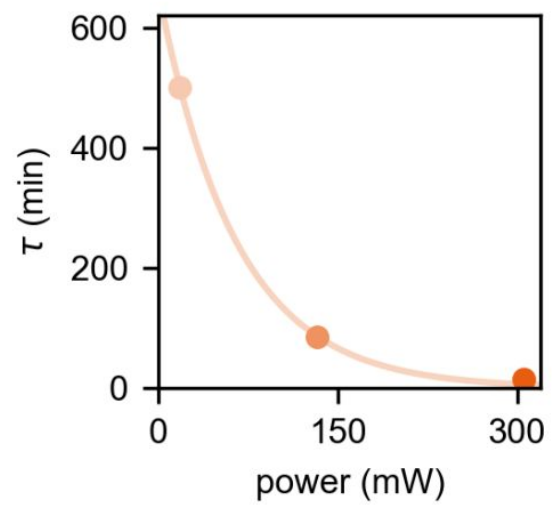

Figure S1 Time constant $\tau$ as a function of laser power. The time constant $\tau$ was determined by fitting the data in Fig. $1 \mathrm{~B}$ with $1-\mathrm{e}^{(-\mathrm{x} / \tau)}$. Note that the response of $\tau$ to increased laser power is non-linear. 

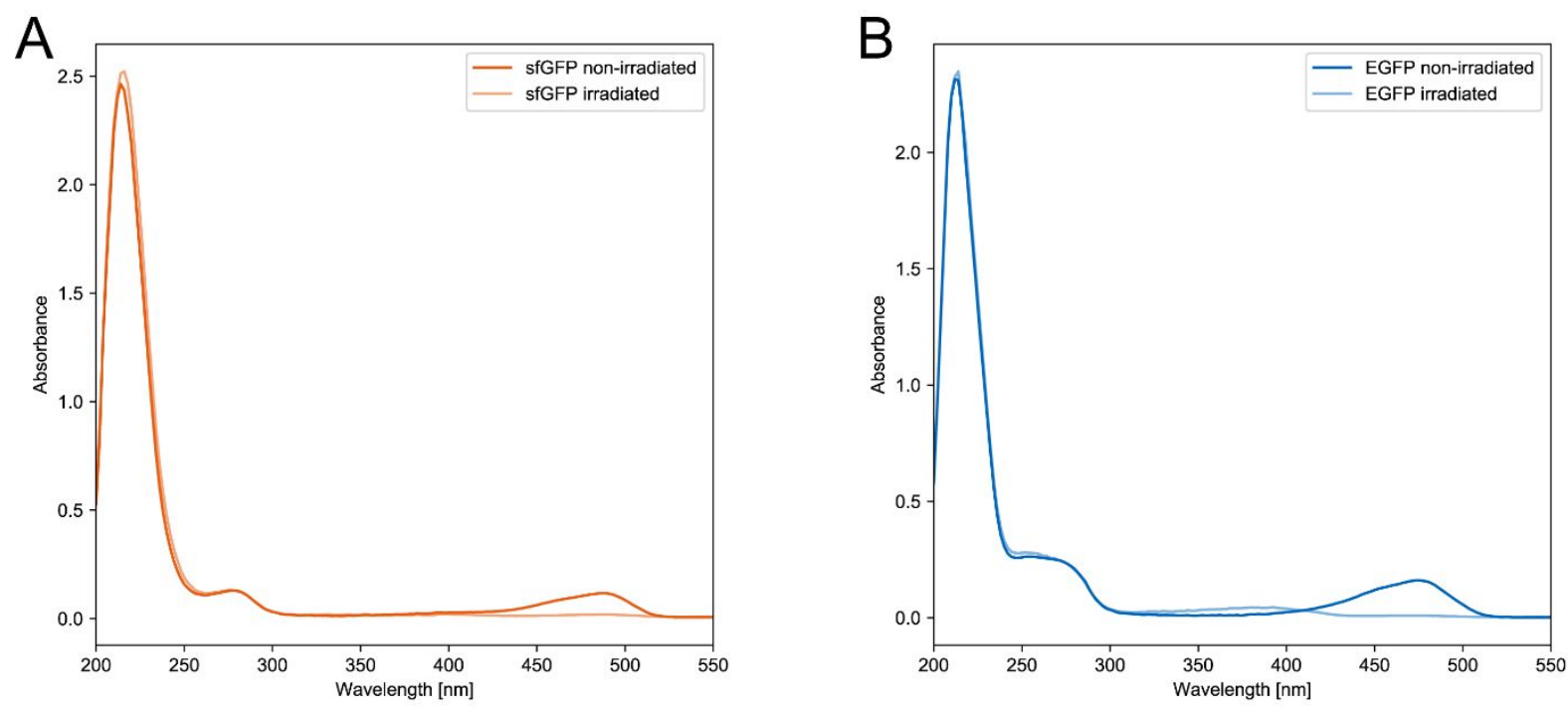

Figure S2 Absorbance spectrum of (A) sfGFP ( $7 \mu \mathrm{M}$ in PBS) and (B) EGFP (14 $\mu \mathrm{M}$ in PBS) before and after irradiation with $445 \mathrm{~nm}$ light. Note the slight increase of EGFP absorption at $\sim 380 \mathrm{~nm}$ after irradiation, suggesting protonation of the chromophore. 
A

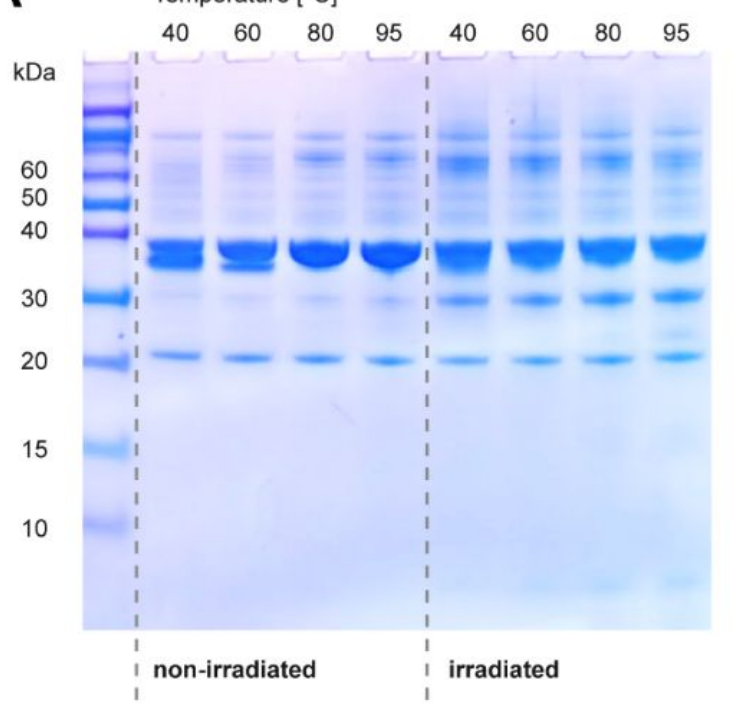

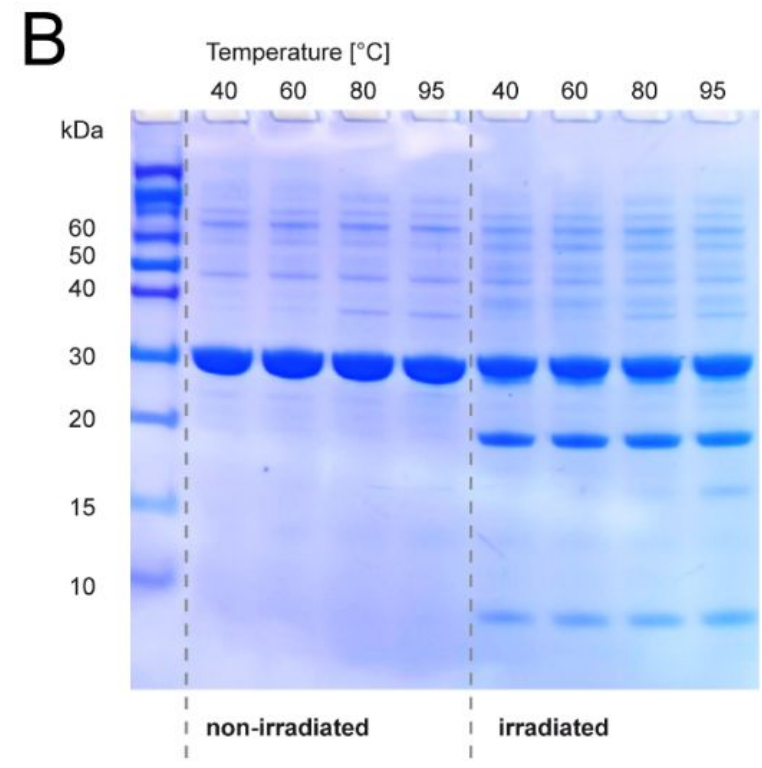

Figure $S_{3}$ Heat treatment of non-irradiated and irradiated GFP samples, analyzed via SDSPAGE. (A) sfGFP ( $7 \mu \mathrm{M}$ in PBS). (B) EGFP (14 $\mu \mathrm{M}$ in PBS). The samples were heated to $40^{\circ} \mathrm{C}$, $60{ }^{\circ} \mathrm{C}, 80^{\circ} \mathrm{C}$, or $95^{\circ} \mathrm{C}$ for 10 minutes prior to SDS-PAGE. Note that formation of the $\sim 31 \mathrm{kDa}$ plus $\sim 8 \mathrm{kDa}$ fragments in the case of sfGFP or the $\sim 22 \mathrm{kDa}$ plus $\sim 10 \mathrm{kDa}$ fragments (EGFP) did not require sample heating. 


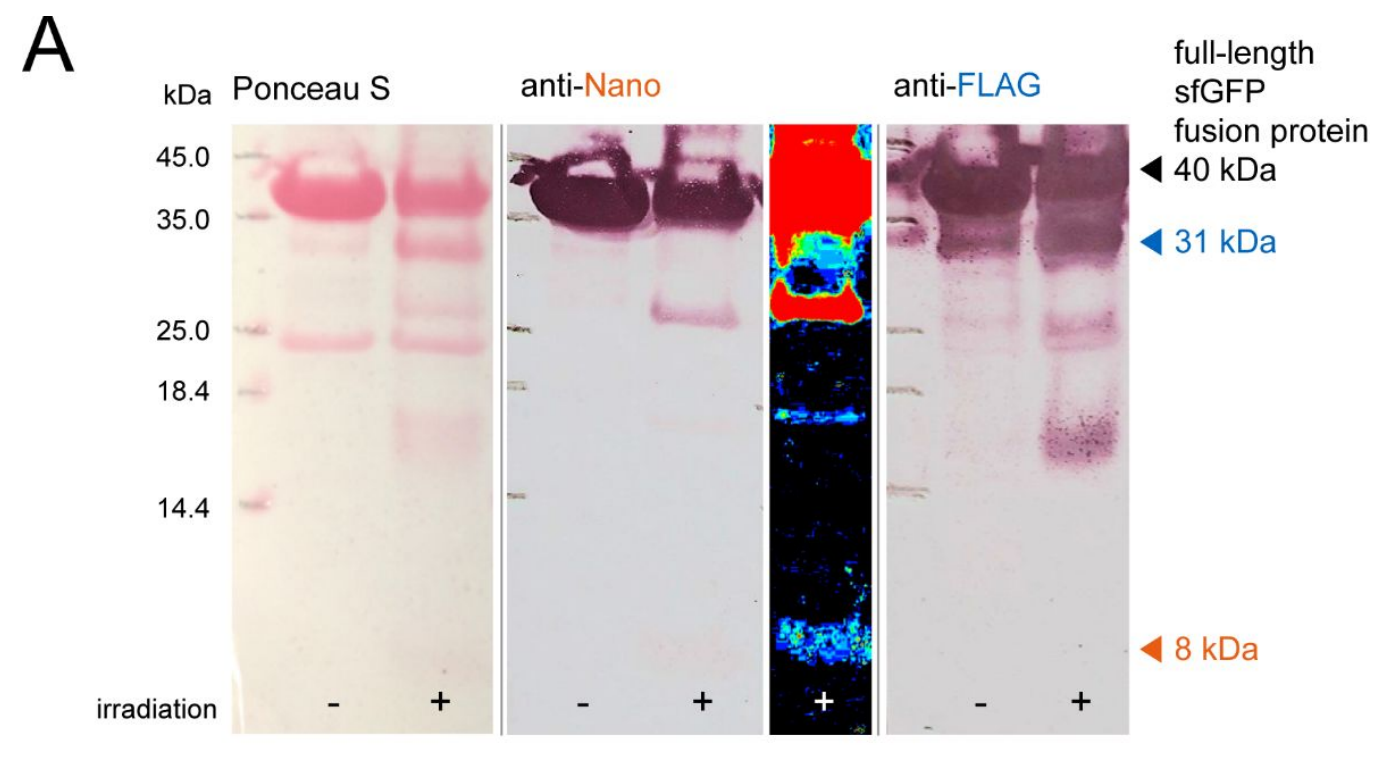

B

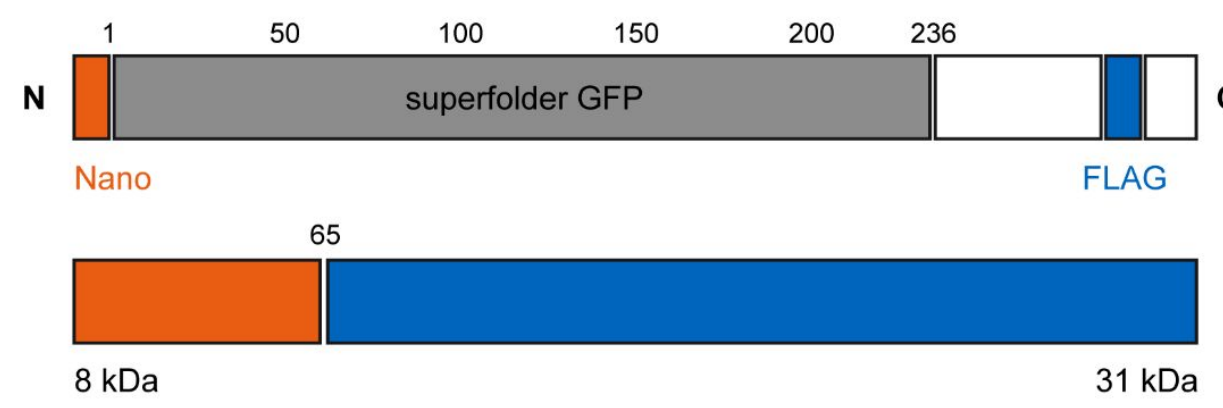

Figure $\mathbf{S}_{4}$ Immunoblot analysis of sfGFP fragmentation. (A) Irradiated and non-irradiated sfGFP were detected by primary antibodies binding an N-terminal Nano-tag or C-terminal FLAG-tag, respectively. For the "anti-Nano + irradiated" segment in this figure, the contrast was increased for the purpose of detecting the $8 \mathrm{kDa}$-terminal fragment. (B) Schematic detailing the makeup of the chimeric sfGFP (upper) and the approximate identification of the cleavage site from the sizes of the fragments (lower). 

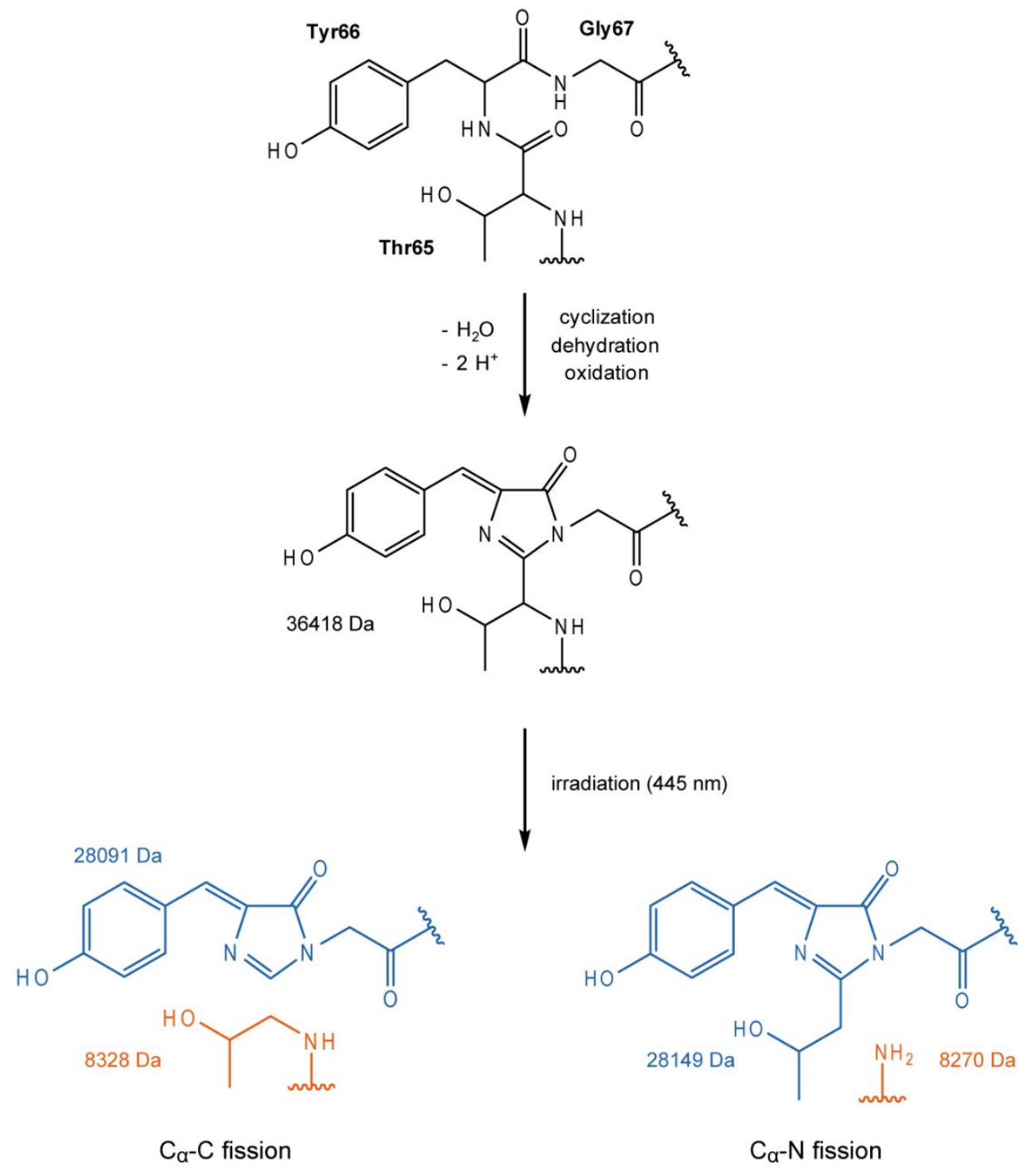

Figure $\mathbf{S}_{\mathbf{5}}$ The postulated sequence of events from the maturation (cyclization, dehydration, oxidation) of the sfGFP fluorophore to its light-induced cleavage. Irradiation might either induce $\mathrm{C}_{\alpha}-\mathrm{C}$ cleavage or $\mathrm{C}_{\alpha}-\mathrm{N}$ cleavage. 


\section{Supporting References}

[1] Heckmeier, P. J., Agam, G., Teese, M. G., Hoyer, M., Stehle, R., Lamb, D. C., and Langosch, D. (2020) Determining the Stoichiometry of Small Protein Oligomers Using SteadyState Fluorescence Anisotropy, Biophys J 119, 99-114.

[2] Cristie-David, A. S., Sciore, A., Badieyan, S., Escheweiler, J. D., Koldewey, P., Bardwell, J. C. A., Ruotolo, B. T., and Marsh, E. N. G. (2017) Evaluation of de novo-designed coiled coils as offthe-shelf components for protein assembly, Mol Syst Des Eng 2, 140-148.

[3] Pedelacq, J. D., Cabantous, S., Tran, T., Terwilliger, T. C., and Waldo, G. S. (2006) Engineering and characterization of a superfolder green fluorescent protein, Nat Biotechnol 24, 79-88.

[4] Schagger, H., and von Jagow, G. (1987) Tricine-sodium dodecyl sulfate-polyacrylamide gel electrophoresis for the separation of proteins in the range from 1 to $100 \mathrm{kDa}$, Anal Biochem 166, 368-379. 